\title{
Every Nearly Idempotent Plain Algebra Generates a Minimal Variety
}

\author{
Keith A. Kearnes * \\ Fachbereich Mathematik, AG1 \\ Technische Hochschule Darmstadt \\ D 64289 Darmstadt \\ Germany
}

An algebra $\mathbf{A}$ is plain if it is finite, simple and has no non-trivial proper subalgebras. An element $0 \in A$ is an idempotent element if $\{0\}$ is a subuniverse and is a non-idempotent element otherwise. A is idempotent if each of its elements is idempotent. In this paper we shall say that $\mathbf{A}$ is nearly idempotent if $\mathbf{A}$ has at least one idempotent element and $\operatorname{Aut}(\mathbf{A})$ acts transitively on the non-idempotent elements.

In [2], Ágnes Szendrei proves that every idempotent plain algebra generates a minimal variety by showing that an idempotent plain algebra with more than two elements generates a congruence modular variety. The proof is not long, but it relies on the classification theorem in [1] for idempotent plain algebras of size $>2$. The proof in [1] of this classification theorem covers several pages. The argument in [2] is completed by directly examining the congruence modular case and the 2-element case and proving for both that an idempotent plain algebra generates a minimal variety. Here we give a short proof of the result using only "V = HSP". With Theorem 4 we show how to boost the result to a proof that every nearly idempotent plain algebra generates a minimal variety.

We say that $\mathcal{V}$ satisfies condition $(E)$ if $\mathcal{V}$ has a unary term $e$ such that for all basic operations $f$ the identity $f(e(x), \ldots, e(x))=e(x)$ holds. If $\mathbf{A}$ is an idempotent plain algebra, then $\mathcal{V}=\mathrm{V}(\mathbf{A})$ satisfies condition $(E)$ with $e(x)=x$.

If $\mathbf{A}$ is plain and $\mathbf{V}(\mathbf{A})$ is not minimal, then there is a plain algebra $\mathbf{B} \in V(\mathbf{A})$ which generates a minimal subvariety. Clearly, $\mathbf{A} \neq \mathbf{B}$ in this case. Szendrei's result can be deduced from the following lemma, since it shows that when $\mathbf{A}$ is plain and idempotent and $\mathbf{B} \in \mathrm{V}(\mathbf{A})$ is plain (and of course idempotent), then $\mathbf{A} \cong \mathbf{B}$.

LEMMA 1 If $\mathbf{A}$ is plain, $\mathcal{V}=\mathrm{V}(\mathbf{A})$ satisfies condition $(E)$ and $\mathbf{B} \in \mathcal{V}$ is idempotent and plain, then $\mathbf{A} \cong \mathbf{B}$.

Proof: Assuming the hypotheses of the lemma we can find $m$, a subalgebra $\mathbf{C} \leq \mathbf{A}^{m}$ and a congruence $\theta$ on $\mathbf{C}$ such that $\mathbf{C} / \theta \cong \mathbf{B}$. Among all such situations, choose one so that $|C|$ is minimal. If $\eta$ is a projection kernel restricted to $\mathbf{C}$ and $\eta \leq \theta$, then $\mathbf{B} \in \mathbf{H}(\mathbf{C} / \eta)=\mathrm{HS}(\mathbf{A})$. $\mathbf{A}$ is plain and $\mathbf{B}$ is nontrivial, so this yields $\mathbf{A} \cong \mathbf{B}$ and finishes the proof. Otherwise, for each projection kernel $\eta$ there is a pair $(a, b) \in \eta-\theta$. We claim that $(e(a), e(b)) \in \eta-\theta$ as well.

Of course, $(a, b) \in \eta$ implies $(e(a), e(b)) \in \eta$. Since $\mathbf{C} / \theta \cong \mathbf{B}$ is idempotent, $e(x) \theta x$ holds on C. Hence $e(a) \theta a$ and $e(b) \theta b$ hold. Now $(a, b) \notin \theta$ implies $(e(a), e(b)) \notin \theta$ by transitivity.

\footnotetext{
* Supported by a fellowship from the Alexander von Humboldt Stiftung.
} 
By condition $(E), e(a)$ is an idempotent element of $\mathbf{C}$. Therefore $D=e(a) / \eta$ is a subuniverse of $\mathbf{C}$ containing $e(a)$ and $e(b)$. Since $\theta$ is nontrivial on $D$ and $\mathbf{B}$ is plain we must have $\mathbf{D} / \theta \cong \mathbf{B}$. By minimality we get $C=D$. Therefore $C$ is a single $\eta$-class for any projection kernel $\eta$. This is impossible since the projection kernels intersect to zero.

Now we begin the proof that every nearly idempotent plain algebra generates a minimal variety. We need two preparatory lemmas.

LEMMA 2 If $\mathbf{A}$ is a nearly idempotent plain algebra, then $\mathrm{V}(\mathbf{A})$ satisfies condition $(E)$.

Proof: Let $U$ be the set of idempotent elements of $\mathbf{A}$ and let $e$ be a unary term of minimal range. Clearly, $e(A) \supseteq U$. If there is an element $u \in e(A)-U$, then the subalgebra generated by $u$ equals $\mathbf{A}$ since $\mathbf{A}$ is plain. In particular, there is a unary term $f$ such that $f(u) \in U$. But now $f e$ has smaller range than $e$ since $f$ collapses two elements of $e(A)$. This contradiction proves that $e(A)=U$. This $e$ satisfies the required identities.

LEMMA 3 Assume that $\mathcal{V}=\mathrm{V}(\mathbf{A})$ where $\mathbf{A}$ is plain, but not idempotent. If Aut $(\mathbf{A})$ acts transitively on the non-idempotent elements of $\mathbf{A}$, then $\mathbf{A} \cong \mathbf{F}_{\mathcal{V}}(1)$.

Proof: Let $a \in A$ be a non-idempotent element. Since $\mathbf{A} \in \mathcal{V}$ it suffices to observe that $\mathbf{A}$ satisfies the universal mapping property with respect to the set $\{a\}$ and some generating class of algebras for $\mathcal{V}$. We take $\{\mathbf{A}\}$ for this generating class. Now any function $f:\{a\} \rightarrow \mathbf{A}$ where $f(a)$ is a non-idempotent element has an extension to some homomorphism $\hat{f}: \mathbf{A} \rightarrow \mathbf{A}$. Simply take an $\hat{f} \in \operatorname{Aut}(\mathbf{A})$ such that $\hat{f}(a)=f(a)$. This extension is unique since $a$ generates A. If instead $f(a)$ is an idempotent element, then the constant map $\hat{f}: \mathbf{A} \rightarrow \mathbf{A}: x \mapsto f(a)$ is the unique extension of $f$ to a homomorphism from $\mathbf{A}$ to $\mathbf{A}$.

THEOREM 4 If $\mathbf{A}$ is nearly idempotent and plain and $\mathbf{B} \in \mathrm{V}(\mathbf{A})$ is plain, then $\mathbf{A} \cong \mathbf{B}$. Hence every nearly idempotent plain algebra generates a minimal variety.

Proof: Together, Lemmas 1 and 2 prove that if $\mathbf{B} \in \mathrm{V}(\mathbf{A})$ is plain, then $\mathbf{A} \cong \mathbf{B}$ or else $\mathbf{B}$ is not idempotent. But if the latter holds and $u$ is a non-idempotent element of $\mathbf{B}$, then $\mathbf{B}=$ $\mathrm{Sg}^{\mathbf{B}}(\{u\})$ is a non-trivial homomorphic image of $\mathbf{A}$ by Lemma 3 , so $\mathbf{A} \cong \mathbf{B}$ holds in this case as well. The arguments in the paragraph preceding Lemma 1 explain why this conclusion proves that every nearly idempotent plain algebra generates a minimal variety.

There is a plain algebra $\mathbf{A}$ which does not generate a minimal variety but whose automorphism group acts transitively on the non-idempotent elements. To construct one such $\mathbf{A}$, begin with the idempotent reduct of a finite, 1 -dimensional vector space and add in all the translations, $x \mapsto x+a$, as new unary operations. Aut $(\mathbf{A})$ contains all the translations and so acts transitively on $A$. If $\theta$ is the kernel of the function $A \times A \rightarrow A:(x, y) \mapsto x-y$, then $(\mathbf{A} \times \mathbf{A}) / \theta$ generates a non-trivial, proper subvariety of $\mathrm{V}(\mathbf{A})$.

There are also plain algebras with idempotent elements which do not generate minimal varieties. Of course, by Theorem 4 any such example must have at least 2 non-idempotent elements. To construct an example, let $A$ be any set which properly contains $\{0,1\}$. Take as basic operations all those operations $p$ on $A$ such that $p\left(A^{n}\right) \neq A$ and $p(w, \ldots, w)=w$ for $w \in A-\{0,1\}$. Then $\mathbf{A}$ is plain and has only two non-idempotent elements. $\mathrm{V}(\mathbf{A})$ is not minimal since the subvariety defined by all the identities of the form $p(\bar{x})=p(\bar{y})$ where $p$ is a basic operation and $\bar{x}, \bar{y}$ are arbitrary tuples of variables is proper and non-trivial. (A non-trivial member of this variety may be constructed as 
a quotient of $\mathbf{A}^{|A|}$.) This paragraph and the preceding one show that neither of the two conditions defining the phrase "nearly idempotent" can be removed if the result of Theorem 4 is to hold.

The paper [3] introduces a class of examples of nearly idempotent plain algebras with exactly one idempotent element. These algebras are used in [4] to provide examples of minimal, locally finite varieties of groupoids which are inherently non-finitely based.

Acknowledgement. We thank the anonymous referee whose suggestions for reorganization substantially shortened the proof.

\section{References}

[1] Á. Szendrei, Idempotent algebras with restrictions on subalgebras, Acta Sci. Math. (Szeged), 51 (1987), 251-268.

[2] Á. Szendrei, Every idempotent plain algebra generates a minimal variety, Algebra Universalis, 25 (1988), 36-39.

[3] Á. Szendrei, Term minimal algebras, to appear in Algebra Universalis.

[4] Á. Szendrei, Nonfinitely based finite groupoids generating minimal varieties, Acta Sci. Math. (Szeged), 57 (1993), 593-600. 